HAEMATOLOGICAL CANCER

\title{
ALL classification-integration of genomic and cytogenetic data
}

Acute lymphoblastic leukaemia (ALL) is characterized by several chromosomal abnormalities that have an important diagnostic and prognostic value, and by numerous genomic copy number alterations with a yet unknown clinical relevance. Anthony Moorman and colleagues have integrated these genomic data with cytogenetic data to refine the genetic classification of paediatric B-cell precursor ALL.

The researchers used a multiplex PCR-based technique to determine the copy number of eight commonly altered genes (IKZF1, CDKN2A/B, $P A X 5, E B F 1, E T V 6, B T G 1$, and RB1) in more than 1,500 patients. "The integration of genomic data in an established cytogenetic classification enabled the identification of two genetic risk groups with different outcome", explains Moorman. The good-risk genetic group included $75 \%$ of children who had excellent response to standard therapy, with a predicted 5-year event free survival and overall survival of $94 \%$ and $97 \%$, and who could be considered for reduced treatment. The second group included $25 \%$ of children with a poor-risk genetic profile, who would benefit from intensive treatment.

The researchers are planning a panEuropean study to validate these findings. "The objective of this research is to further refine and assess the classification so that it can be implemented in the clinic and extend the use of personalized medicine in this disease", concludes Moorman.

\section{Alessia Errico}

Original article Moorman, A. V. et al. A novel integrated cytogenetic and genomic classification refines risk stratification in pediatric acute lymphoblastic leukemia (ALL). Blood doi:10.1182/blood-2014-03-562918 